\title{
Determinación de los parámetros para la reducción de los contenidos de sodio y grasa en la elaboración de salchicha saludable
}

\author{
Determination of parameters for the reduction of sodium and fat \\ contents in the production of healthy sausage \\ Recibido: enero 11 de 2017 | Revisado: marzo 10 de 2017 | Aceptado: abril 15 de 2017
}

\section{Giovanna Castañeda Saavedra ${ }^{\mathrm{I}}$ Resumen $^{-}$}

Mónica Ramos Escudero ${ }^{2}$ El estilo de vida actual ha llevado a la humanidad a Ana María Bernabel Liza ${ }^{3}$ aumentarel consumodeproductoscárnicos procesados; Rosa Aguirre Medrano ${ }^{4}$ sin embargo, esto puede traer consecuencias negativas debido al consumo de grasa y sodio en exceso. El $66 \%$ de los peruanos consume comida rápida, la cual consiste en su mayoría en carnes procesadas, pan y papas fritas, lo cual eleva los niveles de grasa y de sodio por encima de los valores de consumo diario. Por ello, este proyecto tuvo como objetivo elaborar una salchicha cocida tipo Frankfurter, con valores de grasa y sodio por debajo de los valores tradicionales, conservando sus características organolépticas. Este objetivo se logró mediante preparaciones controladas y sus respectivos análisis de control de calidad. El nivel de grasa se redujo desde $15 \%$ hasta $11 \%$, así mismo el contenido de sodio bajó en un $30 \%$ al realizar el análisis por metodologías diferentes

Palabras claves: salchicha Frankfurter, grasa, sodio

\begin{abstract}
The current lifestyles have led humanity to increase the consumption of processed meat products; however, this can bring negative consequences due to the excessive consumption of fat and sodium. 66\% of Peruvians eat fast food, which consists mostly of processed meats, bread and potato chips, which raises fat and sodium levels above daily suggested consumption values. Therefore, this project aims to produce a frankfurter type sausage with values of fat and sodium below the traditional values, preserving its organoleptic characteristics. This objective was achieved through controlled preparations and their respective quality control tests. The level of fat was reduced from $15 \%$ to $11 \%$, and the sodium content was decreased by $30 \%$ by performing the analysis using different methodologies.
\end{abstract}

1 Universidad de San Martín de Porres, Perú mcastanedas@uspe.pe

2 Universidad de San Martín de Porres, Perú mramose@usmp.pe

3 Universidad de San Martín de Porres, Perú abernabell@usmp.pe

4 Universidad de San Martín de Porres, Perú raguirrem@usmp.pe

Key words: fat, sodium, saudage Frankfurter

https://doi.org/10.24265/ campus.2017.v22n23.04

| Campus | Lima, perú | V. XX II | N. 23 | PP. 4I-56 | ENERO-JUNIO | 20I7 | ISSN I8I2-6049 


\section{Introducción}

La grasa animal que se usa para elaborar productos cárnicos emulsionados como las salchichas es muy importante para aportar características de sabor y textura al producto. Sin embargo, el efecto asociado a estas grasas saturadas es precursor de enfermedades cardiovasculares lo cual significa un factor negativo para su consumo. Se han estudiado diferentes alternativas para reducir su contenido en productos cárnicos. Esto modifica las propiedades funcionales de los productos cárnicos emulsionados como el rendimiento, la estabilidad a la cocción y la capacidad de retención de agua, los que tienen efecto sobre el contenido de humedad y rancidez oxidativa, textura y color (Rivera, 2012).

La carne y los productos cárnicos son, generalmente, reconocidos como alimentos altamente nutritivos que proporcionan cantidades notables de proteína (que contiene aminoácidos esenciales para la salud humana), ácidos grasos, vitaminas (es una de las mayores fuentes de vitamina $\mathrm{B}_{12}$ ), minerales (principalmente hierro y zinc) y otros compuestos bioactivos. Sin embargo, también contribuyen a la ingesta de grasas, ácidos grasos saturados (AGS), colesterol, sal y otras sustancias que en cantidades inapropiadas, dependiendo de una amplia variedad de factores, pueden tener consecuencias nefastas para la salud.

Diversos estudios han señalado la existencia de asociaciones entre el consumo de carne y el riesgo de padecer alguna de las enfermedades crónicas no trasmisibles. De hecho, en los últimos años, se han publicado varios estudios resumiendo las evidencias sobre los riesgos del consumo de carnes rojas y las enfermedades cardiovasculares (ECV) ${ }^{10-}$ ${ }^{12}$, el cáncer ${ }^{8,1 C I-15}$, obesidad ${ }^{11-12,16} \mathrm{y}$ diabetes tipo 2 (Wyness, et al, 2011). Un metaanálisis reciente concluyó que el consumo diario de 100 gramos de carne (de cualquier tipo) incrementa el riesgo de cáncer colorrectal entre un 12 a 17\%; mientras que el consumo diario de 25 gramos de carnes procesadas aumenta el riesgo hasta un $49 \%$ (Sandhu, White \& McPherson, 2001).

La hipótesis que se maneja, en la asociación del consumo de carnes rojas y el riesgo de cáncer, se relaciona con la presencia de aminas heterocíclicas mutagénicas e hidrocarbonos aromáticos policíclicos, que se forman durante la cocción a altas temperaturas de este tipo de alimentos. Estos componentes, especialmente, el mutágeno $\mathrm{N}$ nitroso, podría actuar como agente cancerígeno a nivel de la mucosa colorrectal (McCullough y Giovannucci, 2004).

El contenido de sodio de la mayor parte de los alimentos, en su estado natural, es, generalmente, bajo, por esta razón se añade sal a muchos alimentos elaborados. De hecho, las sales de sodio deben estar presentes en la dieta, a fin de reemplazar a las que se pierde en la transpiración. No obstante, el problema ocurre cuando se añade una cantidad excesiva de sal a los derivados procesados de la carne (Fox $\&$ Cameron, 1992). A medida que aumenta el consumo de sal en la dieta, también lo hace la presión arterial. La típica dieta moderna proporciona una cantidad excesiva de sal, desde la infancia hasta la edad adulta. 
La cantidad recomendada de ingesta de sal es inferior a $5 \mathrm{~g} / \mathrm{día} /$ persona. En América, el consumo puede llegar a ser más del doble del nivel recomendado. Todos los grupos etarios, incluidos los niños, se ven afectados. La adición de sal, en la mesa, no es el único problema. En la mayoría de la población, la mayor cantidad de sal en la dieta proviene de los platos preparados y pre-cocinados, incluyendo el pan, carnes procesadas, e incluso cereales para el desayuno (Díaz, 2016). Se ha relacionado el alto consumo de carnes rojas y carnes procesadas con el desarrollo de cáncer, principalmente, de colon y recto (Chao, et al, 2005).

El presente trabajo de investigación permitió elaborar una salchicha funcional con niveles de sodio y grasa menores a las tradicionales. Asimismo, admitió verificar físico-químicamente los niveles de sodio y grasa y comparar con una salchicha tradicional patrón. También se verifican las características organolépticas de la salchicha con un panel de degustación y compararla con la salchicha tradicional patrón; es así como, se determinó la utilidad de la salchicha con reducción de sodio y grasa.

\section{Materiales y Métodos}

\section{Lugar de ejecución}

La ejecución del presente trabajo de investigación se llevó a cabo en los laboratorios de la Escuela Profesional de Ingeniería Industrial de la Facultad de Ingeniería y Arquitectura de la Universidad de San Martín de Porres.

\section{Proceso de elaboración de la salchicha}

- Selección y acondicionamiento de carnes. La materia prima cumple con las características tanto, físicoquímicas como microbiológicas, determinadas de acuerdo con la norma técnica peruana (NTP). La temperatura de almacenamiento de la carne fresca es de 0 a $5^{\circ} \mathrm{C}$.

- Molienda. Las materias primas son molidas en disco de $3 \mathrm{~mm}$.

- Emulsificado. En esta etapa, se realiza la extracción de las proteínas miofibrilares en las mezclas de los insumos cárnicos y no cárnicos, los que se adicionan de acuerdo con el orden secuencial, el mismo que se detalla:

a. Adición de carnes congeladas molidas en disco de $3 \mathrm{~mm}$ en cúter.

b. Adición de sales, sal curantes, fosfatos para la solubilización de las proteínas cárnicas que generan la fuerza iónica de la emulsión más $1 / 3$ de hielo, que se trabaja en cúter.

c. Adición de proteínas vegetales, emulsión de grasa, colorantes, condimentos, conservantes.

d. Trabajar la velocidad del cúter hasta alcanzar una emulsión de $6^{\circ} \mathrm{C}$ y finalmente, adicionar el resto de hielo de la formulación.

e. Agregar las féculas y trabajar hasta alcanzar $12{ }^{\circ} \mathrm{C}$. Luego descargar del cúter.

- Embutición. La pasta fina es embutida en tripas de ovino, calibre 24-26 mm.

- Cocción. Las salchichas se someten a tratamiento térmico hasta alcanzar la temperatura interna $\mathrm{de} 72^{\circ} \mathrm{C}$.

- Enfriado. Las salchichas se enfrían a temperatura ambiente. 




Figura 1. Proceso de elaboración de salchicha cocida de cerdo 
Paso 1. Solubilización de proteínas cárnicas

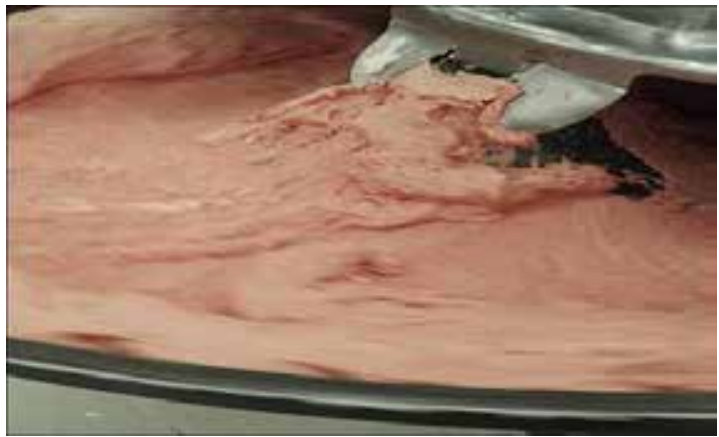

Paso 2. Preparación de la emulsión de grasa con fibras

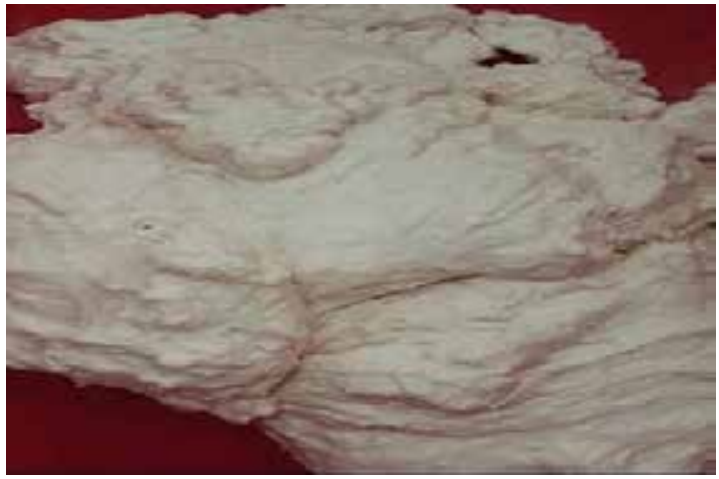

Paso 3. Mezcla de emulsión de grasa con las pastas fina

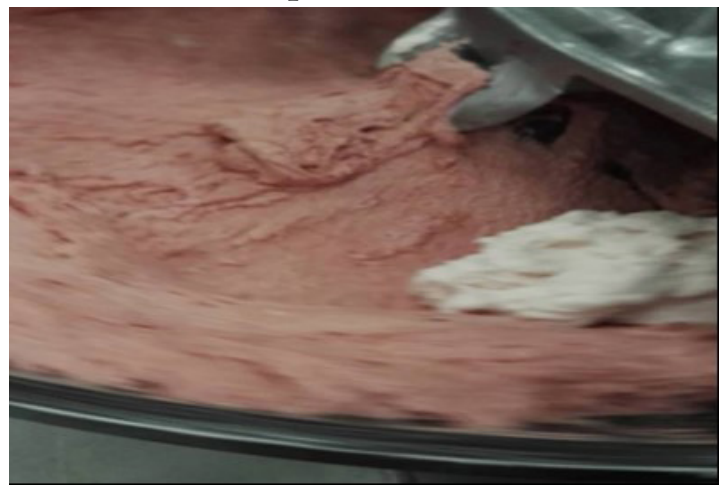

Paso 4. Pasta fina concluida

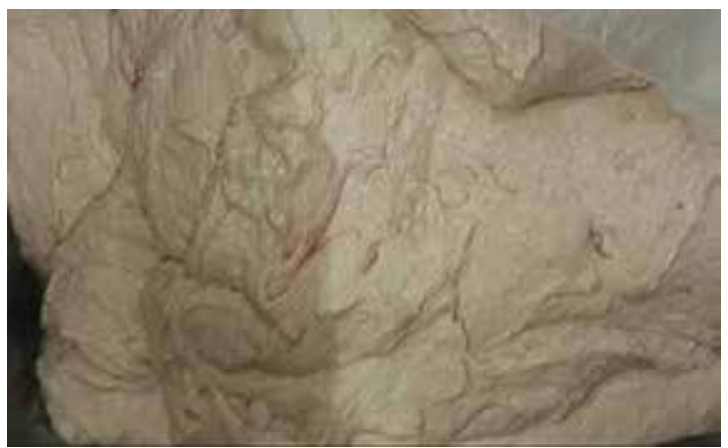

Paso 5. Embutido de salchicha en tripa natural de ovino

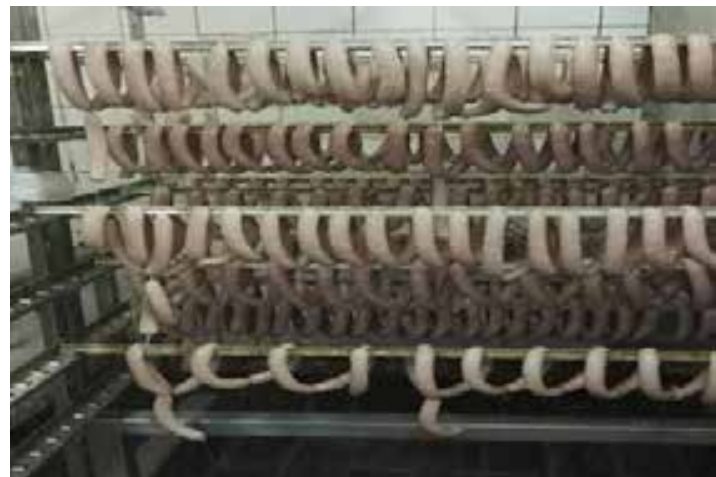

Paso 6. Salchicha cocida y empacada al vacío (producto final)

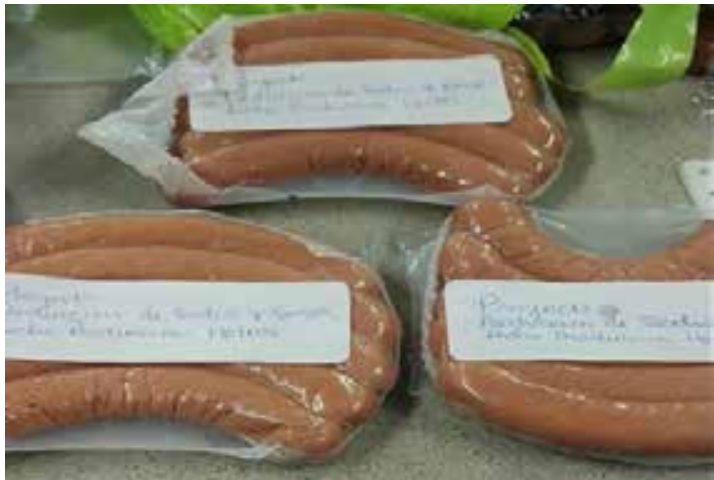

Figura 2. Diagrama de flujo con la secuencia de las operaciones a realizar salchicha con reducción de sodio y grasa

\section{Controles de parámetros y procesa- miento}

La preparación de la salchicha siguió un orden en la adición de los insumos correspondientes en la etapa de proceso.

Primera fase: solubilización de las proteínas miofibrilares de las carnes a las que se añaden sales y fosfatos. Una vez activadas las proteínas, se procedió a la adición de $1 / 3$ de hielo.

Segunda fase: incorporación de proteínas vegetales, colorantes, condimentos, conservantes.

Tercera fase: adición de resto de hielo y finalmente, féculas. La temperatura de salida de la pasta fina es de $12^{\circ} \mathrm{C}$. 


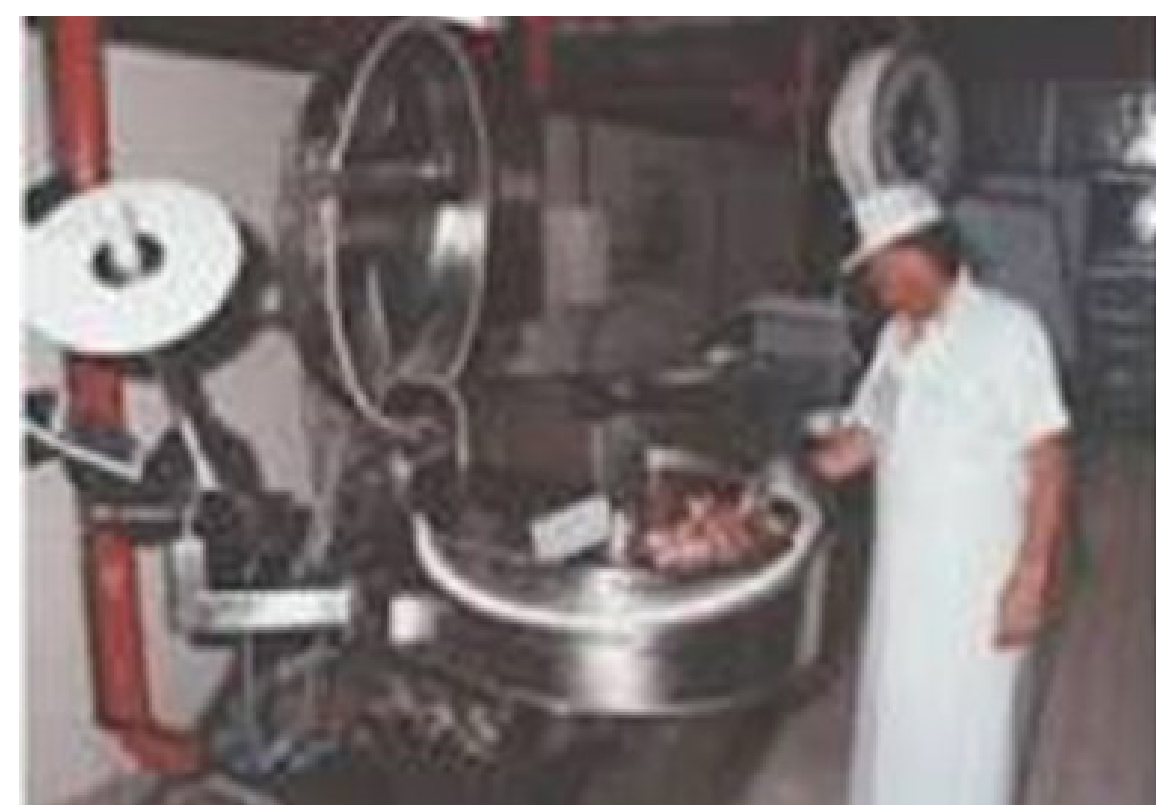

Figura 3. Elaboración de molienda

\section{Formulación de dos tipos de salchichas:}

Tabla 1

Formulación de salchichas

\begin{tabular}{lcc}
\hline & $\begin{array}{c}\text { Formulación } \\
\mathbf{N}^{\circ} \mathbf{1}\end{array}$ & Formulación $\mathbf{N}^{\circ} \mathbf{2}$ \\
\hline \multicolumn{1}{c}{ Ingredientes } & Control & Prueba \\
\hline Recorte de cerdo & $\mathbf{\%}$ & $\mathbf{\%}$ \\
Emulsión de grasa & 57.15 & 57.15 \\
Grasa & 0 & 12 \\
Hielo & 12 & 0 \\
Proteína de soya & 26 & 26 \\
Almidón de yuca & 0.91 & 0.91 \\
Sal & 0.6 & 0.6 \\
Reductor de sal Kcl & 1.36 & 1.04 \\
Azúcar & 0 & 0.32 \\
Sal curante & 0.21 & 0.21 \\
Fosfatos & 0.079 & 0.079 \\
Conservante & 0.22 & 0.22 \\
Antioxidante & 0.45 & 0.45 \\
Condimento & 0.12 & 0.12 \\
Colorante carmín & 0.24 & 0.24 \\
Ajos pelados & 0.06 & 0.06 \\
Cebolla & 0.24 & 0.24 \\
Kion & 0.3 & 0.3 \\
\hline Total & 0.03 & 0.03 \\
\hline & 100.0 & 100.0 \\
\hline
\end{tabular}

Fuente: Elaboración propia 


\section{Controles del producto final}

\subsection{Análisis fisicoquímicos:}

Los análisis físico-químicos comprenden contenido de proteínas, humedad, grasas y contenido de sodio. Esta parte de la investigación tiene dos fases: en la primera parte, se realizó el análisis solo a la salchicha baja en sal y grasa. La segunda parte consistió en realizar el análisis físico químico de las marcas comerciales Braedt, San Fernando, Otto Kunz y la salchicha bajo en sal y grasa.

\subsection{Análisis de proteínas}

Se realizó el análisis para la determinación de proteínas por el método de Kjeldahl, (AOAC 976.06). Se pesó $1,0 \mathrm{~g}$ de muestra en un balón Kjeldahl y se llevó a digestión al equipo, con $\mathrm{CuSO}_{4} \cdot 5 \mathrm{H}_{2} \mathrm{O}$ como catalizador y $\mathrm{K}_{2} \mathrm{SO}_{4}$ como elevador de temperatura. Se le agregó $25 \mathrm{~mL}$ de ácido sulfúrico concentrado y dejó en digestión hasta la disolución total de la muestra (color verde botella). Luego de enfriarla se diluyó con $200 \mathrm{~mL}$ de agua destilada y neutralizó con $\mathrm{NaOH}$ al $50 \%$. Luego se acopló al condensador y encendió el equipo para la destilación. El destilado $(250 \mathrm{ml})$ se recibió en un erlenmeyer de $500 \mathrm{~mL}$ que contiene $50 \mathrm{~mL}$ de ácido bórico al $4 \%$ y el indicador mixto. Concluida la destilación, se lavó el tubo de inmersión y retiró para proceder a la titulación con $\mathrm{HCl} 0,1 \mathrm{~N}$, hasta alcanzar la coloración violeta. Los resultados se expresaron como porcentaje.

\subsection{Análisis de humedad}

El análisis de humedad se llevó a cabo según el método ASTM D3302M ISO 589 , recomendado para alimentos procesados, tipo salchicha. La muestra $(2,0 \mathrm{~g})$ se sometió a $110^{\circ} \mathrm{C}$ en estufa (MARCA) por una hora, se enfrió en el desecador y pesó hasta que el peso fue constante.

\subsection{Análisis de grasa}

Se realizó el análisis de grasas por el método del soxhlet, (AOAC 920.39). Se pesó $2,5 \mathrm{~g}$ de muestra, en un cartucho de papel de filtro, y colocó en la cámara de extracción. En un balón de base plana, se agregó éter de petróleo, luego se armó el equipo soxlet y realizó la extracción, en caliente hasta la extracción total de la grasa. Se la secó en estufa a $65^{\circ} \mathrm{C}$ por una hora, se enfrió y finalmente, pesó. El resultado se expresó en g/100 g.

\subsection{Análisis de sodio}

Para realizar este análisis, se utilizó el Método de Volhard según la norma NC 79-06 para salchichas. Este método se basa en la determinación de cloruros (como cloruro de sodio) presentes en un extracto de la muestra, que ha sido sometida a un tratamiento con agua caliente. Luego de enfriarla se agregó 1 $\mathrm{mL}$ de acetato de zinc y de ferrocianuro de potasio y se llevó a $200 \mathrm{~mL}$. A una parte alícuota del extracto obtenido, se añade solución de nitrato de plata y se valora el exceso con solución de tiocianato de potasio en presencia del indicador férrico. Es un método indirecto para la determinación de cloruros en alimentos. Se precisa que el método se acondicionó de acuerdo con los materiales y reactivos presentes en el laboratorio.

Cálculos. Los cálculos se realizaron a base del contenido de sodio porque este, es uno de los parámetros de control de la salchicha preparada. Se trabajó según la fórmula: 


$$
\operatorname{mg~Na}=\left(\underline{V}_{\underline{A}} * \mathrm{~N}_{\mathrm{A}}-\mathrm{V}_{\underline{B}} * \mathrm{~N}_{\underline{B}}\right) \frac{\mathrm{P}_{\text {meq }}}{\mathrm{w}_{0}} \frac{1000}{10}
$$

Donde:

$\mathrm{V}_{\mathrm{A}}=$ Volumen de nitrato de plata ańadido, en $\mathrm{mL}$

$\mathrm{V}_{\mathrm{B}}=$ Volumen de tiocianato de amonio agregado, en $\mathrm{mL}$

$\mathrm{N}$ = Normalidad de las soluciones

$\mathrm{P}_{\text {meq }}=$ Peso del sodio en $\mathrm{mg}$

$\mathrm{w}_{0}=$ Peso inicial de muestra en gramos

\subsection{Análisis microbiológico}

El análisis microbiológico se efectuó a base de un enfoque del tiempo de vida útil. Este se realizó bajo dos parámetros: el primero es el recuento de microorganimos mesófilos totales (ISO 4833) y el segundo, de bacterias Lacobacillus sp. (ISO 9232).

\subsubsection{Recuento de microrganismos mesófilos totales (ISO 4833)}

Esta determinación indicó el grado de contaminación de una muestra y las condiciones que han favorecido o reducido la carga microbiana. Desde luego, no es aplicable a alimentos fermentados, y ofrece escasa información sobre el manejo del alimento cuando este es poco favorable para el desarrollo microbiano por su $\mathrm{pH}$ o aw, por ejemplo. Este grupo es un indicador importante en alimentos frescos, refrigerados y congelados, en lácteos y en alimentos listos para consumir (RTE por sus siglas en inglés: ready to eat).

Se llevó a cabo a partir de diluciones decimales de la muestra, que se inocularon en placas vertidas de agar triptona glucosa o agar cuenta estándar (PCA). Las placas se incuban en condiciones de aerobiosis, a $35^{\circ} \mathrm{C}$ de 24 a 48 horas. Cifras altas, en este recuento, indican un proceso de alteración del alimento, aunque no necesariamente hay que relacionarlo con la presencia de gérmenes patógenos.

\section{Método I}

1. Se pesó $10 \mathrm{~g}$. de muestra e hizo cuatro diluciones decimales de la misma en AP.

2. Se ańadió con pipetas estériles, a partir de tres de las diluciones decimales $\left(10^{-2}, 10^{-3}, 10^{-4}\right), 1 \mathrm{~mL} /$ placa a una serie de tres placas de Petri vacías estériles. Luego se trabajó la siembra por duplicado.

3. Se vertió en cada placa unos 15 $\mathrm{ml}$ de medio PCA fundido y atemperado de $45-50{ }^{\circ} \mathrm{C}$.

4. A continuación se incubó de 24 h-72 h a $30{ }^{\circ} \mathrm{C}$.

5. Finalmente, se efectuó un recuento de las placas en las que el número de colonias está comprendido entre $30 \mathrm{y}$ 300 y calculó el UFC/g de carne.

\section{Expresión de resultados}

Se retienen las placas que contengan un máximo de 300 UFC al nivel de dos diluciones sucesivas. Es necesario que al menos una placa contenga un mínimo de 15 UFC y calcular el número $\mathrm{N}$ de microorganismos por mililitro o por gramo con la expresión:

$$
N=\frac{\sum C}{\left(n_{1}+0,1 \cdot n_{2}\right) \cdot d}
$$

$\mathrm{N}=$ número de microorganismos por mililitro o por gramo de muestra 
$\Sigma \mathrm{C}$ = suma de las colonias contadas en todas las placas retenidas de dos diluciones sucesivas y con al menos una placa con un mínimo de 15 colonias

$\mathrm{n}_{1}=$ número de placas retenidas en la primera dilución

$\mathrm{n}_{2}=$ número de placas retenidas en la segunda dilución

$\mathrm{d}=$ nivel de dilución correspondiente a la primera dilución retenida $(\mathrm{d}=$ 1, cuando la muestra para análisis se ha sembrado directamente: productos líquidos)

\subsubsection{Recuento de lactobacilos (ISO 9232)}

El método consiste en sembrar un volumen dado de una muestra representativa y homogénea del licuado de salchicha, por analizar y/o diluciones de la misma en placas Petri, utilizando el medio de cultivo MRS, seguido de una incubación anaeróbica a $37^{\circ} \mathrm{C} \pm$ $1^{\circ} \mathrm{C}$ por 72 horas para el conteo de Lactobacillus sp.

Después del período de incubación, se cuentan las colonias características. El número de microorganismos característicos por gramo de muestra se calcula, a partir del número de colonias obtenidas en placas cuyos niveles de dilución muestren un resultado significativo.

\section{Método II}

1. Se pesó $10 \mathrm{~g}$. de muestra y efectuó cuatro diluciones decimales de la misma en agua peptonada.

2. Se añadió con pipetas estériles, a partir de tres de las diluciones decimales $\left(10^{-2}, 10^{-3}, 10^{-4}\right), 1 \mathrm{~mL} /$ placa a una serie de tres placas de Petri vacías estériles y trabajó la siembra por duplicado.
3. A continuación, se vertió en cada placa unos $15 \mathrm{ml}$ de medio MRS fundido y atemperado a $45-50{ }^{\circ} \mathrm{C}$.

4. Posteriormente, se incubó durante $24-72 \mathrm{~h}$, a $30^{\circ} \mathrm{C}$.

5. Finalmente, se efectuó un recuento de las placas entre las que el número de colonias estaba comprendido ( 25 y 250 ) y calculó el UFC/g de carne.

\section{Expresión de resultados}

Retención de las placas que contengan un máximo de 250 UFC al nivel de dos diluciones sucesivas. Es necesario que al menos una placa contenga un mínimo de 25 UFC.

Cálculo del número $\mathrm{N}$ de microorganismos por mililitro o por gramo con la expresión:

$$
N=\frac{\sum C}{\left[\left(1 * n_{1}\right)+\left(0.1 \cdot n_{2}\right)\right] \cdot d}
$$

$\mathrm{N}$ = número de microorganismos por mililitro o por gramo de muestra

$\boldsymbol{\Sigma} \mathrm{C}=$ suma de las colonias contadas, en todas las placas retenidas de dos diluciones sucesivas y al menos una placa con un mínimo de 25 colonias

$\mathrm{n}_{1}=$ número de placas retenidas en la primera dilución

$\mathrm{n}_{2}=$ número de placas retenidas en la segunda dilución

$\mathrm{d}=$ nivel de dilución correspondiente a la primera dilución retenida $(\mathrm{d}=1$ cuando la muestra para análisis se ha sembrado directamente: productos líquidos).

\subsection{Análisis sensorial}

Se tomó una prueba sensorial de aceptación con un panel de 30 consumidores quienes evaluaron la muestra prueba y muestra de prototipo de salchichas comercial. Se 
diseñó la salchicha de cerdo que se elaboró reduciendo la cantidad de sal parcialmente y fue sustituida por $\mathrm{KCl}$. Asimismo, se redujo, parcialmente, la grasa libre presente en la receta y esta fue reemplazada por una emulsión de grasa elaborada con fibras vegetales que fue evaluada, sensorialmente, en una prueba de aceptación de 30 personas que utilizaron la escala hedónica.

\subsubsection{Análisis estadístico}

El procesamiento de la información para los resultados del contenido de proteínas, humedad, grasa, sodio se llevó a cabo a través del programa estadístico SAS, en el cual se determinó la existencia o no de diferencias significativas, a través de un análisis de varianza ANOVA completamente aleatorio y una prueba de Duncan para $\mathrm{P}<0.05$.

Tabla 2

Descripción de los tratamientos en estudio

\begin{tabular}{cl}
\hline Tratamientos & \multicolumn{1}{c}{ Marcas } \\
\hline $\mathrm{T} 1$ & Braedt \\
$\mathrm{T} 2$ & San Fernando \\
$\mathrm{T} 3$ & Otto Kunz \\
$\mathrm{T} 4$ & Salchicha baja en sodio y grasa \\
\hline
\end{tabular}

La evaluación sensorial se analizó mediante la prueba de Friedman. Se realizó la prueba de aceptación con un panel de 30 consumidores, pertenecientes a la población académica de la Facultad de Ingeniería y Arquitectura de la Tabla 3
Universidad de San Martín de Porres. Se aplicó una prueba hedónica de cinco puntos en la que se calificó atributos de olor, sabor, textura, apariencia y color. En esta etapa del estudio, se utilizaron tres muestras de salchichas A, B y SF.

\section{Descripción de los tratamientos en estudio}

\begin{tabular}{clc}
\hline Tratamientos & \multicolumn{1}{c}{ Marcas } & Clave \\
\hline T1 & San Fernando & SF \\
T2 & Salchicha alta en sal y grasa & A \\
T3 & Salchicha baja en sodio y grasa & B \\
\hline
\end{tabular}

\section{Resultados}

\section{Análisis físico- químico}

Los análisis físico-químicos se realizaron solo a la salchicha baja en sal y grasa. El cuadro resumen del análisis físico-químico se puede observar en la Tabla 4:
Tabla 4

Resumen de análisis físico-químico de la salchicha baja en sal y grasa

\begin{tabular}{lc}
\hline \multicolumn{1}{c}{ Determinaciones } & Cantidad (\%) \\
\hline Proteínas & 12.55 \\
Humedad & 69.26 \\
Grasas & 11.29 \\
Cenizas & 3.99 \\
\hline
\end{tabular}


En la Tabla 4, se reportan los resultados obtenidos de los análisis de la salchicha baja en sodio y grasa, los que se interpretan de la siguiente manera:

- Proteínas: de acuerdo con el valor obtenido $12.55 \%$, se ubica como salchicha gourmet.

- Humedad: tiene un valor de 69.26\%.

- Grasa: el nivel de grasa encontrado en la salchicha que es de $11.29 \%$ es más bajo que el reportado para salchichas comerciales en las que se alcanzan valores que oscilan entre 12 y $16 \%$.

- Cenizas: el nivel de cenizas encontrado en la salchicha que es de $3.99 \%$ está dentro de los parámetros normales, que oscilan entre 2.13 y $4.66 \%$.

Los resultados del análisis físicoquímico de las marcas comerciales Braedt, San Fernando, Otto Kunz y la salchicha baja en sal y grasa, se presentan en la Tabla 5.

Tabla 5

Resumen de análisis físico-quimico de salchichas comerciales y la salchicha baja en sal y grasa.

\begin{tabular}{lccccc}
\hline \multicolumn{1}{c}{ Marcas } & Código & Proteínas (\%) & $\begin{array}{c}\text { Humedad } \\
\mathbf{( \% )}\end{array}$ & Grasa (\%) & Sodio (\%) \\
\hline Braedt & $\mathrm{T} 1$ & $12 \pm 0.10^{\mathrm{b}}$ & $72 \pm 0.00^{\mathrm{a}}$ & $15 \pm 0.13^{\mathrm{d}}$ & $344 \pm 0.18^{\mathrm{d}}$ \\
San Fernando & $\mathrm{T} 2$ & $12 \pm 0.20^{\mathrm{b}}$ & $69 \pm 0.10^{\mathrm{c}}$ & $13 \pm 0.23^{\mathrm{c}}$ & $297 \pm 0.19^{\mathrm{b}}$ \\
Otto Kunz & $\mathrm{T} 3$ & $10 \pm 0.10^{\mathrm{c}}$ & $68 \pm 0.10^{\mathrm{d}}$ & $12 \pm 0.20^{\mathrm{b}}$ & $319 \pm 0.24^{\mathrm{c}}$ \\
$\begin{array}{l}\text { Salchicha baja en } \\
\text { sal y grasa }\end{array}$ & $\mathrm{T} 4$ & $13 \pm 0.00^{\mathrm{a}}$ & $70 \pm 0.10^{\mathrm{b}}$ & $12 \pm 0.10^{\mathrm{a}}$ & $100 \pm 0.13^{\mathrm{a}}$ \\
\hline
\end{tabular}

Datos expresados en promedio $\pm S D, n=3$. Valores en una misma columna con diferentes superíndices indican que son diferentes $(\mathrm{p}<0.05)$.

\section{Análisis microbiológico}

En las Tablas 6 y 7, se observan los resultados de los análisis microbiológicos para mesófilos aerobios y Lactobacillus sp., realizados al producto final desde el día 0 hasta el día 56 de almacenamiento para determinar la vida útil del producto.

La Tabla 7 presenta un resumen de todos los análisis microbiológicos realizados al producto, donde también se hallaron la Salmonella sp., Staphilococcus aureus, Escherichia coli y Listeria monocytogenes.

Los resultados del análisis microbiológico, se encuentra dentro de los límites de acuerdo con la norma Criterios microbiológicos R.M. 591 2008 MINSA “Norma Sanitaria”. 
Tabla 6

Número de microorganismos (Aerobios mesofilos) por día evaluado

\begin{tabular}{|c|c|c|c|c|c|c|c|}
\hline DILUCIÓN & Día 0 & Día 7 & Día 28 & Día 35 & Día 42 & Día 49 & Día 56 \\
\hline $10^{-2}$ & 7 & 10 & 11 & 14 & 18 & 27 & 37 \\
\hline $10^{-3}$ & 2 & 4 & 6 & 8 & 12 & 17 & 27 \\
\hline $10^{-4}$ & 0 & 1 & 2 & 4 & 7 & 11 & 19 \\
\hline
\end{tabular}

Tabla 7

Número de microorganismos (Lactobacillus sp.) por día evaluado

\begin{tabular}{|c|c|c|c|c|c|c|c|}
\hline DILUCIÓN & Día 0 & Día 7 & Día 28 & Día 35 & Día 42 & Día 49 & Día 56 \\
\hline $10^{-2}$ & 4 & 6 & 8 & 9 & 9 & 10 & 26 \\
\hline $10^{-3}$ & 16 & 29 & 48 & 76 & 104 & 140 & 166 \\
\hline $10^{-4}$ & 0 & 5 & 19 & 43 & 64 & 107 & 144 \\
\hline
\end{tabular}

Tabla 8

Resultados de análisis microbiológicos de salchicha

\begin{tabular}{|l|c|c|}
\hline \multicolumn{1}{|c|}{ Determinaciones } & 45 días & 60 días \\
\hline Aerobios mesofilos (ufc/g) & $5 \times 10$ & $3 \times 10^{2}$ \\
\hline Escherichia coli (ufc/g) & $<10$ & $<10$ \\
\hline Staphilococcus aureus (ufc/g) & $<10$ & $<10$ \\
\hline Lactobacillus sp. ( ufc/g) & $<10$ & $<10$ \\
\hline Salmonella sp.(/25g) & Ausencia & Ausencia \\
\hline Listeria monocytogenes $(/ 25 \mathrm{~g})$ & Ausencia & Ausencia \\
\hline
\end{tabular}

Fuente: Elaboración propia

\section{Análisis sensorial}

Se aplicó una prueba hedónica de cinco puntos en la que se calificó atributos de olor, sabor, textura, apariencia y color; utilizando la prueba de Friedman. En esta etapa del estudio, se utilizaron tres muestras de salchichas A, B y SF, Tabla 9.

Tabla 9

Descripción de las muestras de salchicha para el análisis sensorial

\begin{tabular}{ccccc}
\hline Tratamientos & Código & Formulaciones & $\begin{array}{c}\text { Sodio } \\
(\mathbf{m g} / \mathbf{1 0 0} \mathbf{g})\end{array}$ & Característica \\
\hline T1 & 197 & $\mathrm{~B}$ & 838,55 & Bajo en sal \\
T2 & 245 & A & 1194,31 & Alto en sal \\
T3 & 300 & San Fernando & 1012,01 & Comercial \\
\hline
\end{tabular}




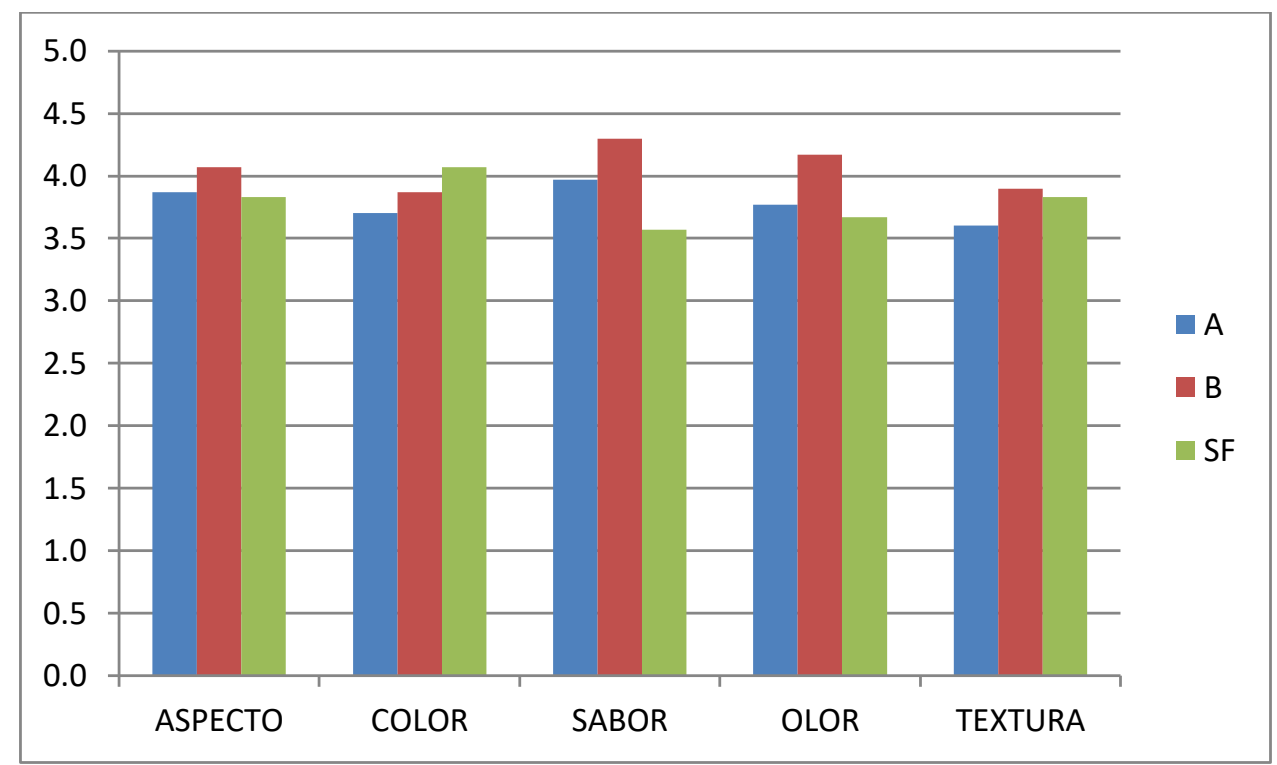

Figura 4. Prueba de aceptación de las tres muestras de salchichas

En la Figura 4, se observan las preferencias de los 30 consumidores en cuanto a los atributos: aspecto, color, sabor, olor y textura. La muestra B (salchicha bajo en sal y grasa) es la más preferida por los panelistas, en relación con las muestras A y SF.

Tabla 10

Prueba de Friedman para los atributos de las tres muestras de salchichas.

\begin{tabular}{lcc}
\hline \multirow{2}{*}{ Atributos } & \multicolumn{2}{c}{ Índice de Friedman } \\
\cline { 2 - 3 } & (Calc.) & (Tab.) \\
\hline Aspecto & 1.425 & 5.991 \\
Color & 2.482 & 5.991 \\
Olor & 3.466 & 5.991 \\
Sabor & 8.865 & 5.991 \\
Textura & 2.220 & 5.991 \\
\hline
\end{tabular}

De acuerdo con la Tabla 10, se expresan los índices de Friedman para los atributos: aspecto, color, olor, sabor y textura. En el aspecto, el índice de Friedman es 1.425, cuyo valor es menor al valor tabulado 5.991, por lo que se rechaza la hipótesis nula, ya que no hay diferencias significativas entre los tres tipos de salchichas. Se da el mismo caso para los atributos de color, olor y textura; excepto para el sabor donde los consumidores sí encontraron diferencias significativas, ya que el índice calculado es mayor al índice tabulado $(8.865>5.991)$ (Tabla 10). Por tanto, la salchicha modificada es más agradable que las salchichas tradicionales.

\section{Discusión}

El propósito fundamental de esta investigación fue ofrecer matrices limpias, saludables y nutritivas. Ello se enmarca en nuestra política de proyección a la comunidad para trabajar el tema, a fin de conservar de la salud del consumidor y la vigencia de los negocios. Por lo tanto, se elaboraron unas salchichas bajas en sodio y grasa a un costo razonable que mantuvieran sus propiedades organolépticas y valor nutricional para beneficio directo del cliente. 
El exceso de sal no se puede eliminar por los rińones. Se acumula en nuestra sangre, atrae el agua e incrementa el volumen de sangre circulante. Esto provoca que el corazón necesite trabajar más para mover la sangre y se eleve la presión produciendo hipertensión arterial, entre otros problemas, tal como lo expresa la Agencia Española de Seguridad Alimentaria y Nutrición (AESAN).

El contenido de humedad, (\%) para el control de calidad de las salchichas, fue en promedio de $69,7 \%$ para las muestras de control y $70,0 \%$ para la muestra del proyecto. El contenido de proteínas, (\%) para el control de calidad en las salchichas, fue en promedio de 12,0\% para la muestra de control y $13.0 \%$ para la muestra del proyecto. El contenido de grasa, para el control de calidad en las salchichas fue en promedio $13,3 \%$ para las muestras de control y $11,3 \%$ para la muestra proyecto.

Con todas estas características, según el Reglamento Técnico de Identidad y Calidad en Salchichas, se trata de una salchicha gourmet de tipo 2.4 .

El contenido de sodio $(\mathrm{mg} / 100 \mathrm{~g})$ para el control de calidad en las salchichas, fue en promedio 320,56 para las muestras de control (Breadt, Otto Kunz y San Fernando) y 251,89 para la muestra proyecto. Considerando que se logró bajar el contenido de sodio en la muestra proyecto, mantuvo los demás valores en un rango razonable.

Respecto al contenido de sodio $(\mathrm{mg} / 100 \mathrm{~g})$, este se determinó por absorción atómica. Se obtuvo $1194,31 \mathrm{mg} / 100 \mathrm{~g}$ en la muestra de la
Breadt; $1012,01 \mathrm{mg} / 100 \mathrm{~g}$ en la de la marca San Fernando y 838,55 mg/100g para la muestra del proyecto, que a pesar de ser valores diferentes a los obtenidos por el método Volhard en el laboratorio, se ve que guardan similar relación.

\section{Conclusiones}

La reducción del contenido de sodio, en productos embutidos, es posible que se considere como el impacto del producto. Las necesidades de funcionalidad de la sal (que actúa con las proteínas de la carne, a través de la capacidad de retención de agua), en la formulación, y los cambios sensoriales esperados en los productos.

El nivel de sodio obtenido en la salchicha fue de $838.55 \mathrm{mg} / 100 \mathrm{~g}$, mientras que en las salchichas comerciales alcanzaron valores entre 1012 y 1194 $\mathrm{mg} / 100 \mathrm{~g}$ de sodio y la reducción de sodio fue de $30 \%$.

Las evaluaciones sensoriales no presentan diferencia significativa de las salchichas comerciales. El nivel de grasa encontrado en la salchicha fue de $11.29 \%$, más bajo que el reportado para salchichas comerciales en las que alcanzaron valores entre 12 y $16 \%$ y su contenido de proteína fue $12.55 \%$, lo que la ubica como una salchicha gourmet.

Las estrategias, en la reducción de grasas y sodio en carnes y derivados cárnicos, son posibles e implican la adherencia a nuevas tecnologías y métodos, el uso de nuevos ingredientes, la adaptación de procesos y la puesta en marcha de ensayos que permitan encontrar el mejor producto, sensorialmente, similar al patrón $y$, nutricionalmente, más saludable. 
La industria de alimentos tiene la responsabilidad social de ofrecer matrices limpias, saludables y nutritivas, es nuestro deber trabajar alrededor del tema para conservar la salud del consumidor y la vigencia de los negocios.

Al respecto, la Organización Mundial de la Salud (OMS) y otras instituciones sanitarias internacionales, recomiendan a la población en general, para una vida sana y sin esfuerzos prolongados, el consumo medio de $5 \mathrm{~g}$ de sal al día, lo que equivale a una cucharadita de sal llena (tamaño de las de café) o $2 \mathrm{~g}$ de sodio al día y que se garantice que sea sal yodada.

\section{AGRADECIMIENTOS}

Al decano de la Facultad de Ingeniería y Arquitectura, Dr. Luis Celi Saavedra por el apoyo que ha prestado en la ejecución del proyecto.

\section{Referencias}

Cámara Argentina de la Industria de Chacinados y Afines - CAICHA. (2009). Reglamento Técnico de Identidad y Calidad en Salchichas. Argentina. Art. 349.

Chao, A., Thun, M., Connel, C., McCullough, M., Jacobs, E., Flanders, D., ..., Calle. E. (2005). Meat consumption and risk of colorectal cancer. JAMA, 293, 172182.

Díaz, A. E. (17 de junio de 2016). Bajo consumo de sal por control de hipertensión. Listín Diario. Recuperado de https:// www.listindiario.co m/lavida/2016/06/16/423469/bajoconsumo-de-sal-por-control-dehipertension

Fox, B.A. \& Cameron, A. G. (1992). Ciencia de los Alimentos, Nutrición y Salud. México: Grupo Noriega Limusa.

McCullough, M. y Giovannucci, E. (2004). Diet and cancer prevention. Oncogene, 23, 6349-6364.

Rivera, R. I. (2012). Fat reduction and alternatives for its substitution in emulsified meat products, a review. NACAMEH, 6(1), 1-14.

Sandhu, M. S., White, I. R. \& McPherson, K. (2001). Systematic review of the prospective cohort studies on meat consumption and colorectal cancer risk: a metaanalytical approach. Cancer Epidemiol Biomarkers Prev., 10, 439 - 446.

Wyness, L., Weichselbaum, E., O'Connor, A., Williams, E. B., Benelam, B., Riley, H., \& Stanner, S. (2011). Red meat in the diet: an update. Nut Bull, 36, 34-77. 
